\title{
Therapeutic Approaches to Insomnia and Fatigue in Patients with Multiple Sclerosis
}

This article was published in the following Dove Press journal:

Nature and Science of Sleep

\author{
Michelle Drerup' \\ Alicia Roth' \\ Alexa Kane 2 \\ Amy B Sullivan (D) ${ }^{2}$ \\ 'Sleep Disorders Center, Cleveland \\ Clinic, Cleveland, OH, USA; ${ }^{2}$ Mellen \\ Center for MS, Cleveland Clinic, \\ Cleveland, OH, USA
}

Correspondence: Michelle Drerup Cleveland Clinic, 9500 Euclid Ave S-73,

Cleveland, $\mathrm{OH}, 44195$, USA

Tel + I 216445925 |

$\mathrm{Fax}+12166360090$

Email drerupm@ccf.org

\begin{abstract}
The prevalence of sleep disorders in individuals with multiple sclerosis (MS) is 3-5 times higher compared to the general population. Insomnia Disorder, defined as difficulty falling asleep, maintaining sleep or waking up too early, can lead to significant fatigue, the most common and disabling symptom of MS. In addition, fatigue and insomnia in patients with MS also can overlap with and exacerbate other psychological and physical symptoms. Cognitive behavioral therapy for insomnia (CBT-I) has been shown as an effective treatment for chronic insomnia and burgeoning research has demonstrated the effectiveness of this treatment for insomnia in individuals with a variety of comorbid medical conditions including MS. The purpose of the current review will explore the literature surrounding the prevalence and impact of sleep disorders and fatigue in MS. Additionally, this review will address practical ways to help individuals with MS manage fatigue as well as how to modify typical standard behavioral treatments for insomnia to take into account special considerations for individuals with MS based on the level of disability and other comorbid issues that impact sleep.
\end{abstract}

Keywords: CBT-I, sleep, comorbidity, neurological disorder, multiple sclerosis

\section{Introduction}

Insomnia Disorder is defined as difficulty falling asleep, staying asleep, or early morning awakenings with greater than three months duration and associated with impairment in daytime functioning. ${ }^{1}$ The prevalence of Insomnia Disorder varies depending on the diagnostic nosology utilized. Based on the International Classification of Diseases 10th Edition (ICD-10), prevalence is approximately $4 \%$; if based on the International classification of sleep disorders second edition (ICSD-2) criteria, 15\%; and if based on the Diagnostic and Statistical Manual of Mental Health Disorders fourth edition text revision (DSM-IV) criteria is $22 \%{ }^{2}$

Previous research suggests insomnia is highly prevalent amongst persons with medical conditions. Taylor et $\mathrm{al}^{3}$ found that a range of diagnoses were associated with a high prevalence of insomnia in a community population, including breathing problems (prevalence of insomnia: 59.6\%), gastrointestinal problems $(55.4 \%)$, chronic pain (48.6\%), hypertension (44\%), and urinary problems $(41.5 \%)$.

The definition of insomnia and its clinical relevance has evolved to reflect the complexity of determining the etiology of sleep disturbances. Insomnia was previously defined as either primary or secondary. Primary insomnia occurred when insomnia was not attributable to another medical or mental health condition. Secondary insomnia referred to insomnia that was a symptom of a medical or 
mental health disorder. In 2005, the National Institutes of Health's State of the Science Conference on Insomnia solidified the term "co-morbid insomnia." Previously, insomnia co-occurring with a medical or psychiatric disorder was called "Other Insomnia Due to a Known Physiological Condition" or "Other Insomnia Due to a Mental Disorder"4 Co-morbid insomnia better encapsulated the reciprocal nature of sleep complaints and chronic health or mental health conditions.

This distinction reflects a more modern conceptualization of the development of Insomnia Disorder and its chronicity. The 3-P Model of Insomnia suggested by Spielman et $\mathrm{al}^{5}$ proposes that Insomnia Disorder develops due to predisposing, precipitating, and perpetuating factors. Predisposing factors are risk factors associated with sleep disturbance (e.g., age, genetics, mental health history). Precipitating factors are triggers that lead to symptoms of insomnia. Perpetuating factors are the maladaptive behaviors, cognitions, or environmental factors that maintaininsomnia; in other words, coping mechanisms adopted to manage poor sleep that is actually prolonging insomnia.

The re-conceptualization of secondary insomnia has been valuable in developing interventions for persons with co-morbid insomnia and medical conditions. Utilizing the 3-P model to develop these interventions means that the co-existing medical problem does not need to be cured or improved for sleep to improve, despite the co-morbid condition being the precipitating factor.

At present, Cognitive Behavioral Therapy for Insomnia (CBT-I) is the first-line treatment for Insomnia Disorder recommended by the American College of Physicians. ${ }^{6}$ CBT-I targets maladaptive behaviors, attitudes, beliefs, and thought patterns that perpetuate insomnia (see Figure 1 for details of components of CBT-I). CBT-I has been shown to be an effective intervention for insomnia across numerous chronic medical conditions. For example, McCrae et $\mathrm{al}^{6}$ found that both CBT-I and Cognitive Behavioral Therapy for Pain (CBT-P) produced significant improvements in sleep, though the effects of CBT-I were of greater magnitude and sustained longer. In female breast cancer survivors postcancer treatment, CBT-I was also effective in improving the amount of time to fall asleep, sleep efficiency, subjective insomnia, and physical and cognitive functioning compared to placebo. ${ }^{7}$ In post-menopausal women with insomnia, CBT-I and Sleep Restriction therapy alone (a single component of CBT-I) decreased subjective insomnia; CBT-I also accounted for significantly greater reductions in time awake in the middle of the night. ${ }^{8}$ Crawford et al $^{9}$ found that $94.3 \%$ of women with chronic migraine who completed a digital CBT-I course responded to treatment (i.e., $\geq 50 \%$ reduction in insomnia symptoms). And a pilot study examining the effects of brief behavioral therapy for insomnia (BBT-I) for persons with irritable bowel syndrome showed that BBT-I improved sleep quality, insomnia severity, and sleep efficiency compared to a placebo intervention. ${ }^{10}$ As a whole, the literature suggests that successfully improving insomnia in persons with chronic medical conditions is possible without directly intervening on the medical condition itself.

The purpose of the current review is to explore the extant literature on the prevalence of sleep disturbance and fatigue in persons with Multiple Sclerosis (PwMS). Additionally, this review will introduce practical modifications to existing cognitive behavioral treatments for insomnia and fatigue that have been tailored for PwMS by experts in both behavioral sleep medicine and MS.

\section{Sleep and Fatigue Prevalence in Patients with MS}

Sleep disturbance or poor sleep quality in PwMS is common and often under-recognized and inadequately addressed. Previous research suggests the prevalence of sleep disturbance in the MS population is between $48 \%$ and $67 \%$ and up to four times higher in PwMS as compared to the general population. ${ }^{11-14}$ A recent study found that over $70 \%$ of PwMS screened positive for having at least one sleep disorder although only $13 \%$ indicated having a diagnosis of a sleep disorder. ${ }^{15}$ More specifically, insomnia, obstructive sleep apnea (OSA), restless leg syndrome (RLS) and circadian rhythm disorders are among the most common sleep disorders in the general population as well as the MS population. ${ }^{15}$

There are no large-scale epidemiological studies of the true prevalence of Insomnia Disorder in MS, but it is generally accepted that it is a common problem that affects approximately $40-50 \%$ of patients. ${ }^{12}$ In a recent study, it was reported that $42 \%$ of MS patients had difficulty in initiating sleep, 53\% reported frequent awakenings, and 58\% reported wake after sleep onset. ${ }^{16}$ OSA prevalence in MS is not well established. Limited studies have reported values from $4 \%{ }^{17}$ to as high as $58 \%$. ${ }^{18}$ The prevalence of RLS in MS patients is approximately $20 \%$ and some studies show a three to five times higher prevalence in patients with MS. ${ }^{19-21}$ The prevalence of circadian rhythm sleep disorders is not known; however, it has been suggested circadian rhythm sleep disorder is higher in PwMS with severe fatigue. ${ }^{22}$ 
Why We Sleep \& Sleep Stages

What Controls Sleep:

- Circadian Rhythms

- Sleep Drive/Pressure

Spielman 3-P Model of Insomnia

- Predisposing, Precipitating, Perpetuating Factors

\section{Stimulus Control}

Addresses conditioning between bed \& wakefulnes Bed becomes strong trigger for sleep

Wake up and get out of bed

the same time every day,

even on weekends or nonwork days

Go to bed when you are sleepy, but not before your goal bedtime.

Avoid napping during the day

Use the bed for sleep and intimacy only.

Get up when you can't sleep.

\section{Sleep Hygiene}

Stop Caffeine between $12-$

$2 \mathrm{PM}$

\section{Stop nicotine 2 hrs before} bedtime

No exercise 2 hrs before bedtime

Sleep Hygiene recommendations are necessary but insufficient

Sleep Hygiene recommendations are nece
to treat insomnia
No heavy meals 2 hrs before bedtime (but don't go to bed hungry)

No electronics in the bedroom (TV, phone)

Keep bedroom cool, dark, quiet

\section{Sleep Restriction}

Restricting TIME IN BED based on total sleep time

Don't worry or plan in bed

Do not try too hard to sleep!

Based on Patient's Sleep

Diary Data

Create a buffer zone. and wake times

\section{Relaxation}

\begin{tabular}{|l|l|}
\hline \multicolumn{1}{|c|}{ Relaxation } \\
\hline \multicolumn{1}{|c|}{$\begin{array}{c}\text { Reduces physical arousal at bedtime } \\
\text { Reinforcing bed as relaxing and place for sleep }\end{array}$} \\
\hline $\begin{array}{l}\text { Diaphragmatic Breathing } \\
\text { Mindfulness }\end{array}$ & Guided Imagery \\
\hline $\begin{array}{l}\text { Progressive Muscle } \\
\text { Relaxation }\end{array}$ & Autogenic Training \\
\hline & Don't worry or plan in bed \\
\hline Encourage to practice during the day to decrease overall \\
physical hyperarousal
\end{tabular}

Figure I Components of cognitive behavioral therapy for insomnia (CBT-I).

Sleep disturbances in PwMS, as described above, are due to several pathophysiology factors which include demyelinating lesions in the brainstem and spinal cord. Demyelination is a central feature of MS as the myelin
Increases sleep pressure \& eliminates time in bed awake (reduces conditioning)

- Allow pt to choose bed Example:

Anchor wake time \& $\quad$ TST: 6 hrs

$\begin{array}{lll}\text { count backwards } & \text { Sleep Restriction }=6 \mathrm{hrs}\end{array}$

Important:

- Cannot compensate for sleep loss

- Have to consistently stick to wake up time

\section{Cognitive Therapy}

Identify \& address myths/maladaptive thoughts about sleep and cognitive hyperarousal at bedtime

Identify maladaptive beliefs about sleep

- "I read that everyone needs at least 8 hours of sleep"

- "I won't sleep at all without my Ambien"

- "I can't function on the sleep I get now"

- "8 hours is the average, but everyone's sleep need is different"

Challenge maladaptive thoughts \& myths to improve emotional experience of

- "I may have trouble sleeping without Ambien, but now I have the tools to naturally sleep"

insomnia

- "I might not sleep well tonight, but I will still be able to work tomorrow and take care of my kids" sheath that protects nerves is stripped off during inflammation periods. Lesions in the brainstem and spinal cord are known to contribute to difficulty with breathing control, upper airway muscle activity and unstable ventilator 
drive which would contribute to central sleep apnea and possibly insomnia. ${ }^{23}$ Additionally, it has been demonstrated that sleep supports oligodendrocytes functions, including myelination, and repair processes after white matter damage. ${ }^{23}$ These repair processes increase during sleep but are reduced after sleep deprivation. ${ }^{23}$ Therefore, it is likely that poor sleep quality can have a negative influence on remyelination processes and possible recovery after an MS relapse. In a recent study, patients with relapsing-remitting MS and poor sleep quality were more likely to have an increased rate of relapses for a longer duration which supports the theory that poor sleep may ultimately impair the ability to repair from MS relapses. ${ }^{23}$ This underscores the importance of assessing and treating sleep disorders in PwMS.

Fatigue can be defined as an overwhelming sense of lack of physical and/or mental energy that interferes with usual or desired activities. ${ }^{24}$ Fatigue is a common, multidimensional, and complex symptom of MS and impacts up to $90 \%$ of PwMS at some point during the course of the disease. Additionally, 55\% of PwMS report fatigue as the worst symptom. $^{15,24,25}$ Fatigue significantly impedes on PwMS daily life, personal relationships and work quality. A recent study found $66 \%$ of PwMS reported fatigue limited daily functioning, 37\% reported it limited social functioning and $61 \%$ reported fatigue limited functioning at work. ${ }^{26}$ While the exact cause of MS-related fatigue is still unknown, sleep disturbances are suggested to be one of the largest predictors of fatigue in MS when examined among disease variables and depression. ${ }^{27}$

Fatigue is a significant symptom of MS and can affect multiple activities of daily living, work status, social and family life and quality of life. ${ }^{28}$ PwMS often describe "invisible symptoms" (not outwardly visible) as the most debilitating and difficult to manage, which includes fatigue, as well as pain, sexual dysfunction, cognitive issues and mood symptoms. These symptoms are often described as debilitating, frustrating, aggravating and difficult to treat by both patients and providers. Fatigue is a subjective and physical experience and is often described by PwMS as "having a 24/7 flu," "never feeling rested," or "exhausting and debilitating."

\section{Tailoring Insomnia Treatment for Patients with MS}

Promising research has found significant improvements in symptoms of insomnia with Cognitive Behavioral Therapy for Insomnia (CBT-I) in PwMS. A retrospective study of
11 individuals with MS who underwent CBT-I found that $86 \%$ experienced a reduction in insomnia symptoms, $60 \%$ reported a reduction in fatigue, and $73 \%$ reported increased total sleep time. ${ }^{29}$ Similarly, a case study of an individual with MS reported improvements in sleep quality, life satisfaction, anxiety and depression after a brief course of CBT-I. ${ }^{30}$ Based on these initial promising data, Siengsukon et $\mathrm{al}^{31}$ conducted the first prospective randomized control trial $(n=33)$ and demonstrated that CBT-I is feasible in PwMS and produced improvements in insomnia severity, sleep quality, sleep self-efficacy, as well as improvements in comorbid symptoms of fatigue, depression and anxiety.

In terms of the practical application of these strategies, exploration of potential modifications to standard strategies and special considerations based on symptoms and needs of this population are advisable. Cognitive impairments that are associated with MS may serve as a barrier to various aspects of CBT-I. One of the key components of CBT-I is having the patient complete a sleep log to track sleep patterns and response to treatment. Individuals with MS who experience cognitive difficulties may need assistance from a caregiver or aide to complete the sleep logs. Another option may be to assess patients' sleep patterns by having them use a clinical-grade actigraph or commercially available sleep tracker. In addition, written materials with detailed descriptions of the patient's treatment plan would be helpful for both patients and their caregiver(s) who may be facilitating the implementation of the sleep plan.

Specific perpetuating factors to consider when modifying CBT-I in PwMS include individuals spending an excessive amount of time in bed due to physical and motor limitations. Implementing stimulus control guidelines may be difficult or impossible depending on the severity of MS symptoms. For example, a stimulus control guideline is to delay getting into bed until sleepy to increase sleep drive and minimize the length of time until sleep onset. This strategy may not be possible for individuals who rely on the assistance of a caregiver or aide to get into bed. Another guideline of stimulus control is that the individual should get out of bed if they are not falling asleep within 20-30 minutes. This may not be feasible due to physical limitations or may actually cause too much effort/exertion and actually lead to increased alertness and arousal. Developing an alternative plan with the individual to make a distinction of being in bed relaxing/winding down versus actual bedtime/trying to 
sleep may include things like changing position in bed from sitting upright to laying down or changing cues in the environment to clearly signal bedtime (eg, turning off TV and lights, setting book down on nightstand).

Exploring MS-related causes of the middle of the night awakenings need to be taken into including bladder problems and muscle spasticity. For nocturia, one strategy that may help would be to have individual try to discontinue liquid consumption 2-3 hours before bedtime. If necessary, a consult to urology may be beneficial and consideration of certain medications to decrease urinary frequency. Muscle spasticity is also a common symptom of MS and can be very uncomfortable and lead to more disrupted sleep. Oftentimes exercise and stretching can help reduce spasticity and medications are also available to reduce discomfort and decrease the impact on sleep.

Sleep restriction is a strategy that increases sleep pressure and enhances conditioning between the bed and sleep. Time in bed is limited to an individual's average sleep time. For example, if someone is only sleeping an average of 6 hours and their desired wake time in the morning is $6 \mathrm{am}$, their recommended sleep schedule would be to delay bedtime until 12 am and have a consistent wake time of 6 am. For patients with chronic health issues, sleep compression is a modified version of sleep restriction that involves a gradual, step-wise reduction of time in bed until the target total sleep time is reached. For a person with 6 hours of total sleep time and 10 hours of in bed time, sleep compression would involve reducing their time in bed by 30 min every week. Although completely avoiding naps is recommended in the standard application of CBT-I and sleep restriction, high levels of fatigue in PwMS may make naps necessary and beneficial. For patients in which naps are considered essential, smarter napping should be discussed including keeping naps time limited (20-30 mins), in their own bed, and preferably earlier in the daytime. Combining CBT-I with additional fatigue management strategies would also likely be extremely beneficial for many PwMS.

\section{Fatigue Management Interventions}

Fatigue is a significant symptom of MS, affecting approximately $80 \%$ of PwMS. ${ }^{32}$ Despite advances in both pharmacological and non-pharmacological treatment, fatigue is difficult to manage. Assessment can also be complicated as the mechanisms of action are unknown; however, several factors that impact fatigue in PwMS have been identified. These may include primary factors such as immune dysregulation, central nervous system mechanisms, endocrine factors, and neurotransmitter dysregulation. Secondary factors may include: physical deconditioning, sleep dysfunction, pain, psychological factors, depression, and medications. ${ }^{32}$ In addition, practitioners and researchers suggest that PwMS who have complaints of fatigue should undergo an appropriate evaluation to rule out a sleep disorder precipitating or perpetuating the MSrelated fatigue as well as depression and/or anxiety. ${ }^{22,32}$ For instance, MS-related fatigue may mask the existence of a sleep disorder or fatigue and a sleep disorder such as insomnia may be comorbid. In 2004, Attarian et $\mathrm{al}^{33}$ compared 15 PwMS who reported fatigue to 15 PwMS who did not present with fatigue. Interestingly, 12 out of the 15 individuals with fatigue reported disrupted sleep or disrupted circadian rhythm as compared to 3 out of the 15 without fatigue.

Research suggests that although more investigation is necessary to fully understand fatigue and fatigue interventions, we currently understand the need for a multidisciplinary long-term management including both pharmacological and non-pharmacological treatments. Khan et $\mathrm{al}^{32}$ provided a comprehensive review of the literature with detailed suggestions on fatigue management in PwMS. A summary of these includes: rehabilitation therapies which include exercise and strength building, aquatic therapies, behavioral and educational interventions, and possibly pharmacological intervention. Rehabilitation interventions are also recommended to manage MS-related fatigue, and several studies have demonstrated that interventions such as exercise, energy conservation management (pacing) can have positive short-term effects on fatigue outcomes. ${ }^{34-36}$

Managing fatigue in clinical practice comes with an appropriate assessment to rule out other medical or psychological conditions. If it is determined that it is fatigue related to MS, treatment can be tailored to the person's needs. Approaches to fatigue management are generally centered on the identification and development of activity modifications that reduce fatigue through systematic analysis. ${ }^{37}$ With regards to behavioral and educational interventions, pacing is likely the most commonly used strategy. Pacing is the act of matching tasks to ability and energy reserves and can include having PwMS complete a daily activity, fatigue and mood log to determine when they feel they are least and most fatigued. ${ }^{38}$ This often reveals patterns to help PwMS understand their body as well as the timing of their energy levels. This technique includes breaking tasks down into smaller parts, building in long or short rest periods and monitoring energy levels 
and needs for breaks (Werfel, 2016). Additionally, assisting PwMS find a balance between what is desirable and what is possible highlights the importance of pacing in optimizing the quality of life. Additionally, in order to assist patients in understanding how to manage fatigue, the body energy bank analogy is frequently used. Patients are taught that in order to have energy, one must replenish energy as well as add to energy. This means appropriate and restorative sleep, increasing energy by movement, if possible, and contributing energy by looking at relaxation strategies. Mental health and cognitive disorders can also impact fatigue (e.g., energy expended with rumination, anxiety, depression, cognitive decline and excessive concentration). Managing fatigue is an important and necessary component for contributing to the best quality of life for each patient. Assessment to determine their source or sources of fatigue is vital as is tailoring treatment to each individual person. ${ }^{38}$

\section{Discussion}

Although there is an abundant literature on the prevalence of sleep disorders and fatigue in persons with MS, there is a dearth of empirical findings on managing these conditions for PwMS. The standard applications of first-line treatments may be inappropriate for PwMS given their physical or cognitive limitations; however, addressing poor sleep and fatigue is crucial in this population. Despite the few studies examining interventions for insomnia with PwMS, results from the extant literature show that modifications to CBT-I that are tailored for PwMS can be successful. ${ }^{31}$ Similarly, researchers and clinicians have modified fatigue interventions developed for rehabilitation to appropriately address fatigue for PwMS.

Additionally, studies that have investigated sleep in chronic medical problems have also noted reciprocity between insomnia improvement and improvement in medical symptoms. ${ }^{6,10}$

Similarly, Baron et $\mathrm{al}^{39}$ found that insomnia symptoms were improved when PwMS underwent psychotherapy targeted for depression. Hence, improving sleep and fatigue may be an opportunity to improve mental health or physical symptoms of MS as well.

That sleep and fatigue can be improved for PwMS is promising evidence for the quality of life for PwMS. That insomnia and fatigue can be considered co-morbid conditions with MS instead of secondary to MS means that there is potential to improve sleep and daytime functioning despite the progressive nature of MS. Specific aspects of or behaviors related to MS can be targeted as perpetuating factors for insomnia and fatigue that make standard interventions tailored for this special population.

\section{Abbreviations}

BBT-I, Brief Behavioral Therapy for Insomnia; CBT-I, Cognitive Behavioral Therapy for Pain; DSM-IV, CBT-P Diagnostic and Statistical Manual of Mental Health Disorders fourth edition text revision; ICD-10, International Classification of Diseases 10th Edition; ICSD-2, International classification of sleep disorders second edition; MS, Multiple Sclerosis; OSA, obstructive sleep apnea; PwMS, Person(s) with Multiple Sclerosis; RLS, restless leg syndrome.

\section{Disclosure}

Dr Amy Sullivan reports personal fees from Novartis, Genentech, National MS Society, and Dynamed, outside the submitted work. The authors report no other potential conflicts of interest in this work.

\section{References}

1. American Psychiatric Association. Diagnostic and Statistical Manual of Mental Disorders. 5th ed. 2013.

2. Roth T, Coulouvrat C, Hajak G, et al. Prevalence and perceived health associated with insomnia based on DSM-IV-TR; international statistical classification of diseases and related health problems, tenth revision; and research diagnostic criteria/international classification of sleep disorders, second edition criteria: results from the America Insomnia Survey. Biol Psychiatry. 2011;69(6):592-600. doi:10.1016/ j.biopsych.2010.10.023

3. Taylor DJ, Mallory LJ, Lichstein KL, Durrence HH, Riedel BW, Bush AJ. Comorbidity of chronic insomnia with medical problems. Sleep. 2007;30(2):213-218. doi:10.1093/sleep/30.2.213

4. NIH State-of-the-Science Conference. Statement on manifestations and management of chronic insomnia in adults. NIH Consens State Sci Statements. 2005;22(2):1-30.

5. Spielman AJ, Caruso LS, Glovinsky PB. A behavioral perspective on insomnia treatment. Psychiatr Clin North Am. 1987;10(4):541-553. doi:10.1016/S0193-953X(18)30532-X

6. McCrae CS, Williams J, Roditi D, et al. Cognitive behavioral treatments for insomnia and pain in adults with comorbid chronic insomnia and fibromyalgia: clinical outcomes from the SPIN randomized controlled trial. Sleep. 2019;42(3). doi:10.1093/sleep/zsy234

7. Matthews EE, Berger AM, Schmiege SJ, et al. Cognitive behavioral therapy for insomnia outcomes in women after primary breast cancer treatment: a randomized, controlled trial. Oncol Nurs Forum. 2014;41 (3):241-253. doi:10.1188/14.ONF.41-03AP

8. Cl D, Da K, Jt A, et al. Treating chronic insomnia in postmenopausal women: a randomized clinical trial comparing cognitive-behavioral therapy for insomnia, sleep restriction therapy, and sleep hygiene education. Sleep. 2019;42(2). doi:10.1093/sleep/zsy217

9. Crawford MR, Luik AI, Espie CA, et al. Digital cognitive behavioral therapy for insomnia in women with chronic migraines. Headache. 2020;60(5):902-915. doi:10.1111/head.13777 
10. Ballou S, Katon J, Rangan V, et al. Brief behavioral therapy for insomnia in patients with irritable bowel syndrome: a Pilot Study. Dig Dis Sci. 2020;65(11):3260-3270. doi:10.1007/s10620-020-06182-w

11. Garland SN, Scurrey SRM, Ploughman M. Health, lifestyle and aging with MS Canadian consortium. Factors associated with poor sleep in older adults with multiple sclerosis. Int J Behav Med. 2017;24 (6):937-945. doi:10.1007/s12529-017-9653-4

12. Sakkas GK, Giannaki CD, Karatzaferi C, Manconi M. Sleep abnormalities in multiple sclerosis. Curr Treat Options Neurol. 2019;21(1):4. doi:10.1007/s11940-019-0544-7

13. Bamer A, Johnson K, Amtmann D, Kraft G. Prevalence of sleep problems in individuals with multiple sclerosis. Mult Scler. 2008;14 (8):1127-1130. doi:10.1177/1352458508092807

14. Boe Lund HM, Aae TF, Indrevåg W, et al. Poor sleep in patients with multiple sclerosis. PLoS One. 2012;7:11. doi:10.1371/journal. pone.0049996

15. Braley TJ, Boudreau EA. Sleep disorders in multiple sclerosis. Curr Neurol Neurosci Rep. 2016;16(5):50. doi:10.1007/s11910-016-0649-2

16. Alhazzani AA, Alshahrani A, Alqahtani M, et al. Insomnia among non-depressed multiple sclerosis patients: a cross-sectional study. Egypt J Neurol Psychiatr Neurosurg. 2018;54:1. doi:10.1186/ s41983-018-0016-0

17. Brass SD, Li C-S, Auerbach S. The underdiagnosis of sleep disorders in patients with multiple sclerosis. J Clin Sleep Med. 2014;10 (9):1025-1031. doi:10.5664/jcsm.4044

18. Kaminska M, Kimoff RJ, Benedetti A, et al. Obstructive sleep apnea is associated with fatigue in multiple sclerosis. Mult Scler. 2012;18 (8):1159-1169. doi:10.1177/1352458511432328

19. Manconi M, Ferini-Strambi L, Filippi M, et al. Multicenter Case-Control Study on restless legs syndrome in multiple sclerosis: the REMS Study. Sleep. 2008;31(7):944-952. doi:10.5665/sleep/31.7.944

20. Li Y, Munger KL, Batool-Anwar S, De Vito K, Ascherio A, Gao X. Association of multiple sclerosis with restless legs syndrome and other sleep disorders in women. Neurology. 2012;78 (19):1500-1506. doi:10.1212/WNL.0b013e3182553c5b

21. Auger C, Montplaisir J, Duquette P. Increased frequency of restless legs syndrome in a French-Canadian population with multiple sclerosis. Neurology. 2005;65(10):1652-1653. doi:10.1212/01. wnl.0000184519.47689.c4

22. Najafi MR, Toghianifar N, Etemadifar M, Haghighi S, Maghzi AH, Akbari M. Circadian rhythm sleep disorders in patients with multiple sclerosis and its association with fatigue: a case-control study. $J$ Res Med Sci. 2013;18(Suppl 1):S71-S73.

23. Buratti L, Iacobucci DE, Viticchi G, et al. Sleep quality can influence the outcome of patients with multiple sclerosis. Sleep Med. 2019;58:56-60. doi:10.1016/j.sleep.2019.02.020

24. Capone F, Collorone S, Cortese R, Di Lazzaro V, Moccia M. Fatigue in multiple sclerosis: the role of thalamus. Mult Scler. 2020;26 (1):6-16. doi:10.1177/1352458519851247

25. Miller P, Soundy A. The pharmacological and non-pharmacological interventions for the management of fatigue related multiple sclerosis. J Neurol Sci. 2017;381:41-54. doi:10.1016/j.jns.2017.08.012
26. Iriarte J, Subirá ML, Castro P. Modalities of fatigue in multiple sclerosis: correlation with clinical and biological factors. Mult Scler. 2000;6(2):124-130. doi:10.1177/135245850000600212

27. Strober LB. Fatigue in multiple sclerosis: a look at the role of poor sleep. Front Neurol. 2015;6. doi:10.3389/fneur.2015.00021

28. Haselkorn J, Balsdon Richer C, Fry Welch D. Multiple sclerosis council for clinical practice guidelines. Overview of spasticity management in multiple sclerosis. Evidence-based management strategies for spasticity treatment in multiple sclerosis. J Spinal Cord Med. 2005;28(2):167-199. doi:10.1080/10790268.2005.11754545

29. Clancy M, Drerup M, Sullivan AB. Outcomes of cognitive-behavioral treatment for insomnia on insomnia, depression, and fatigue for individuals with multiple sclerosis. Int J MS Care. 2015;17(6):261-267. doi:10.7224/1537-2073.2014-071

30. Majendie C, Dysch L, Carrigan N. Cognitive Behavioral Therapy for Insomnia (CBT-I) for an adult with multiple sclerosis. Clin Case Stud. 2017;16(2):115-131. doi:10.1177/1534650116674594

31. Siengsukon CF, Alshehri M, Williams C, Drerup M, Lynch S. Feasibility and treatment effect of cognitive behavioral therapy for insomnia in individuals with multiple sclerosis: a pilot randomized controlled trial. Mult Scler Relat Disord. 2020;40:101958. doi:10.1016/j.msard.2020.101958

32. Khan F, Amatya B, Galea M. Management of fatigue in persons with multiple sclerosis. Front Neurol. 2014;5. doi:10.3389/fneur.2014.00177

33. Attarian HP, Brown KM, Duntley SP, Carter JD, Cross AH. The relationship of sleep disturbances and fatigue in multiple sclerosis. Arch Neurol. 2004;61(4):525-528. doi:10.1001/archneur.61.4.525

34. Blikman LJ, Huisstede BM, Kooijmans H, Stam HJ, Bussmann JB, van Meeteren J. Effectiveness of energy conservation treatment in reducing fatigue in multiple sclerosis: a systematic review and meta-analysis. Arch Phys Med Rehabil. 2013;94(7):1360-1376. doi:10.1016/j.apmr.2013.01.025

35. Asano M, Finlayson ML. Meta-analysis of three different types of fatigue management interventions for people with multiple sclerosis: exercise, education, and medication. Mult Scler Int. 2014;2014. doi:10.1155/2014/ 798285

36. van den Akker LE, Beckerman H, Collette EH, Eijssen ICJM, Dekker J, de Groot V. Effectiveness of cognitive behavioral therapy for the treatment of fatigue in patients with multiple sclerosis: a systematic review and meta-analysis. $J$ Psychosom Res. 2016;90:33-42. doi:10.1016/j.jpsychores.2016.09.002

37. Guidelines, Multiple Sclerosis Clinical Practice. Fatigue and Multiple Sclerosis: Evidence-Based Management Strategies for Fatigue in Multiple Sclerosis. Paralyzed Veterans of America; 1998.

38. Werfel P, Franco Duran R, Trettin L. (2016). Multiple Sclerosis: Advances in psychotherapy - evidence-based practice. Cambridge, MA: Hogrefe \& Huber.

39. Baron KG, Corden M, Jin L, Mohr DC. Improvement in insomnia with psychotherapy for depression in patients with multiple sclerosis. J Behav Med. 2011;34(2):92-101. doi:10.1007/s10865-010-9288-2

Nature and Science of Sleep

Dovepress

\section{Publish your work in this journal}

Nature and Science of Sleep is an international, peer-reviewed, open access journal covering all aspects of sleep science and sleep medicine, including the neurophysiology and functions of sleep, the genetics of sleep, sleep and society, biological rhythms, dreaming, sleep disorders and therapy, and strategies to optimize healthy sleep.
The manuscript management system is completely online and includes a very quick and fair peer-review system, which is all easy to use. Visit http://www.dovepress.com/testimonials.php to read real quotes from published authors. 